\title{
Reconstruction Algorithm and Switching Graph for Two-Projection Tomography with Prohibited Subregion
}

\author{
Akira Kaneko ${ }^{1, \star}$ and Rina Nagahama ${ }^{2}$ \\ ${ }^{1}$ Department of Information Sciences, Ochanomizu University, \\ 2-1-1, Otsuka, Bunkyo-ku, Tokyo, 112-8610, Japan \\ kanenko@is.ocha.ac.jp \\ http://www.is.ocha.ac.jp/ kaneko/ \\ ${ }^{2}$ Graduate School of Humanities and Sciences \\ Ochanomizu University \\ rinax@atom.is.ocha.ac.jp
}

\begin{abstract}
We consider the two-projection tomography problem, assuming a priori known prohibited region. We show that a modification of Ryser's reconstruction algorithm gives a solution. We then study the relation of the switching graph for the solution sets with and without the prohibited region. Finally, we apply our idea to get a better reconstruction figure imposing prohibited region artificially.
\end{abstract}

\section{Introduction}

We consider the reconstruction problem of a discrete plain figure $F$ contained in a rectangle $I$ from its two projections $f_{y}(x)$ and $f_{x}(y)$ along the $y$ - and $x$ axis, respectively. This is equivalent to finding a binary matrix from its columnand row-sums, but we prefer the geometric notation better related with the continuous tomography. See [11] for general reference on this problem. In this report we assume that $F$ is a priori known to have no building cell (that is, filled with 0 for the binary matrix formulation) in a subregion $J$ of $I$, and consider the reconstruction problem with this constraint.

We show that under the assumption of uniqueness for $J$ Ryser's algorithm for the reconstruction without constraint can be modified to obtain a solution of this problem. Then we study the structure of the solution set by means of a graph, extending our former work [7] for the full solution set without constraint.

Then we apply our idea to obtain a better solution of the reconstruction problem without constraint, by setting artificial constraint as a priori knowledge. This works faster than the strategy of successive improvement adopted so far. In the final section we try characterization for the prohibited region.

The reconstruction problem with rectangular constraint was considered by Brualdi and Dahl [3]. In [4] an equivalent result for unique figure $J$ is announced

\footnotetext{
^ Partially supported by Grant-in-Aid for Scientific Research No. 16540140.
} 
without proof. We hope that our reconstruction algorithm is simple and practical. As further related works, Fulkerson [5] considered reconstruction of binary square matrices with zero diagonal, which is a typical non-unique figure. Also, Kuba [10] studied problem of reconstruction with prescribed 1's, which is intimately related with the present problem, but not equivalent (see Remark 1 (2) of $\S 2$ ). The work of Anstee [1] can also be understood of reconstruction with prescribed 1's. We thank the referee for informing us these references.

\section{Setting of the Problem and Reconstruction Algorithm}

Let $I$ denote the rectangular region $a \leq x<b, c \leq y<d$ in the first quadrant of $\boldsymbol{R}^{2}$, where $a, b, c, d \in Z$. Let $F$ be a subregion of $I$ which is the union of integer cells $C_{i j}:=[i, i+1) \times[j, j+1)$. We shall denote its characteristic function also by $F$. Thus its $y$-projection $f_{y}(x)$, or $x$-projection $f_{x}(y)$ is defined as

$$
f_{y}(x)=\int_{c}^{d} F(x, y) d y, \quad f_{x}(y)=\int_{a}^{b} F(x, y) d x .
$$

These take integer values, representing the number of cells in the respective columns or rows. The reconstruction problem from the two projections is to find $F$ from the projection data $f=\left\{f_{y}(x), f_{x}(y)\right\}$. Define the arrangements by

$$
\begin{aligned}
& f_{x y}(x)=\operatorname{meas}\left\{y ; f_{x}(y) \geq x\right\}, \quad f_{y x}(y)=\operatorname{meas}\left\{x ; f_{y}(x) \geq y\right\}, \\
& f_{y x y}(x)=\operatorname{meas}\left\{y ; f_{y x}(y) \geq x\right\},
\end{aligned}
$$

where meas denotes the one-dimensional length. In the discrete case, this is equivalent (modulo measure 0) to the permutation of the columns or rows in decreasing order and finally view all from the $x$-axis. Then the consistency condition, that is, the condition for the existence of a solution, given by Lorentz, Gale and Ryser is

$$
\forall x \quad \int_{0}^{x} f_{x y}(t) d t \geq \int_{0}^{x} f_{y x y}(t) d t, \quad \text { and } \quad \int_{0}^{\infty} f_{x y}(t) d t=\int_{0}^{\infty} f_{y x y}(t) d t,
$$

The uniqueness of the solution is assured if and only if the equality holds for all $x$ in the first inequality above. For further information about this problem see the survey article [11. We here recall only necessary materials.

First we review Ryser's reconstruction algorithm in a form given in [6]. We shall call this hereafter the Ryser-Kaori algorithm without constraint.

1. Choose the tallest column from $f_{y}(x)$. In case of tie, choose the leftmost one.

2. Remove this column from $f_{y}(x)$ and at the same time, remove the same number of cells from $f_{x}(y)$ one for each row by the strategy of the longest row first.

3. Modify the graph of $f_{x}(y)$ crashing the removed cells toward the $y$-axis.

4. Return to 1 if there still remain cells in the projection data. 
Now we have a subregion $J \subset I$ where we should not place any cell in reconstruction. In what follows we assume that $J$ constitutes a unique figure, that is, there is no other figure having the same projection data. Note the following.

Lemma 1. $J$ is unique if and only if by a permutation of columns and rows it is brought to the form of Lorentz's renormalization of the projections, that is, to the form of union of height-decreasing adjacent subrectangles with the lower edge common to that of I (see Figure 1) :

$$
J=J_{1} \cup J_{2} \cup \cdots \cup J_{r} .
$$

In fact, the sufficiency is obvious. For the necessity, we can obviously find a permutation bringing the $y$-projection $f_{y}(x)$ to the monotone decreasing form. Then by the assumption of uniqueness, the $y$-projection $f_{x y}(x)$ of the $x$-projection $f_{x}(y)$ agrees with this. Since the $y$-projection is achieved by a permutation of rows, after these two permutations the $y$ - and $x$-projection of $J$ agree with this monotone figure. In view of the uniqueness, $J$ itself has the same form.

Thus, we shall assume henceforth without loss of generality that $J$ has the above form (2).

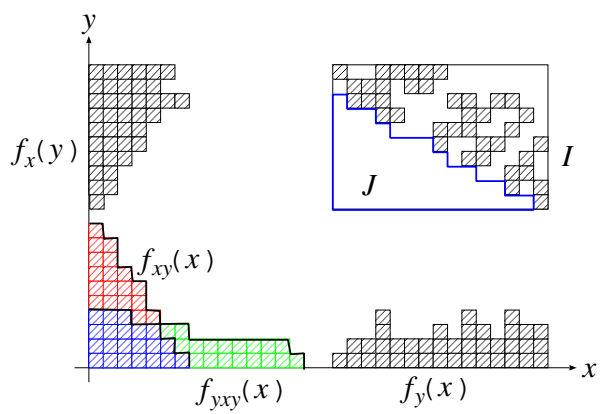

Fig. 1. Figure with prohibited region and its projections

Now we explain the reconstruction algorithm. We assume that the projection data are $J$-consistent, that is, there exists at least a solution with $J$ as prohibited region.

1. For $i=1$ to $r$ do

2. Choose the tallest column from $f_{y}(x)$ among those above $J_{i}$. In case of tie, choose the leftmost one.

3. Remove this column from $f_{y}(x)$ and at the same time, remove the same number of cells from $f_{x}(y)$ one for each level by the strategy of the longest level first, but among the rows not touching $J_{i}$.

4. Modify the graph of $f_{x}(y)$ crashing the removed cells.

5. Return to 2 if there still remain cells above $J_{i}$.

6. End for. 
Theorem 1. The above algorithm successfully gives a reconstruction figure which does not contain any cell in $J$, provided that the projection data are $J$-consistent.

Proof. We proceed by the induction of the total number of cells in the figure. The case of one cell is trivial. Assume that the assertion is true for any $J$ and for any $J$-consistent projection data up to $n$ cells, and consider a problem with $n+1$ cells. By the assumption of $J$-consistency, there exists a solution figure $F$ which we may not know concretely. Following the above algorithm, we first choose the tallest column among those above $J_{1}$. For each cell in this column, we pick up a cell from $f_{x}(y)$ at the longest row not touching $J_{1}$. If the chosen cell exists in the figure $F$, we are correctly diminishing the data. If we chose a cell, say $P$, from a row where there was no cell in that column of the figure $F$, then there should exist a cell of $F$, say $P^{\prime}$, in the column of $P$ which was not chosen by the reconstruction algorithm. On the other hand, $P$ comes from a cell of $F$, say $Q$, in another column by $x$-projection, There are several candidates of such $Q$, but we claim that among them there is at least one such that $P^{\prime}$ and $Q$ constitute a switching component in $F$, that is, the place $R$ in Figure 2 is vacant. In fact, if all the counterparts in the row of $P^{\prime}$ are occupied by the cells of $F$, then the $x$-projection at the row of $P^{\prime}$ will have length greater than that at the row of $P$. This violates the rule of algorithm that we should pick up the cell from the longer rows of $f_{x}(y)$ first. This argument applies to all cells chosen in relation to this column. Thus after removing the column from $f_{y}(x)$ and the corresponding cells from $f_{x}(y)$ there remain projection data which come from a true figure $F^{\prime}$ obtained by several switching as mentioned above from $F$. This means that the remaining projection data are $J$-consistent, and by the induction hypothesis, we can obtain a solution of reconstruction with the constraint. By adding the first treated column to this solution, we obtain a solution for given size.

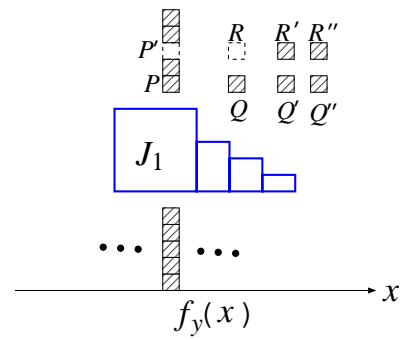

Fig. 2. Proof of justification of reconstruction algorithm

The converse is obvious: if our algorithm ends up using all the cells in the projection, we obtain a reconstruction with the given constraint $J$. Thus it presents a practical criterion for the $J$-consistency.

Remark 1. (1) The direct application of Ryser-Kaori algorithm, that is, processing from the tallest of all columns ignoring $J$, does not work. Figure 3 is such an example. 
(2) One may think of an alternative algorithm such as filling the prohibited region by cells and applying Ryser-Kaori algorithm without constraint, then removing the cells in the prohibited region will give a desired solution. But in general it is not easy to obtain a reconstruction of which the prohibited region is filled with cells. See, however, $§ 5$ in this respect.

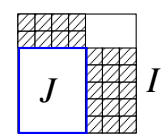

Fig. 3. Necessity of modification of reconstruction order

\section{Switching Graph}

It is well known that a figure is non-unique, namely, there is another figure with the same $y$ - and $x$-projections, if and only if it contains a switching component. For a non-unique figure we can relate a graph to the solution set by considering each solution as a vertex, and connecting a pair of solutions by an edge if and only if they are transformed by one switching operation. This graph seems to have been first introduced by Brualdi 2 under the name of interchange graph. Later, 9] re-intruduced it and called Ryser graph. Ignoring these, we called it the switching graph and studied its properties with many examples. Further we gave a direction to each edge showing the type modification from type 2 to type 1 (see Figure 4), thus producing the switching digraph ([7]). Since we employ in the sequel permutation of columns and rows which may change the direction of engaged edges, we only consider the switching graph in this paper.

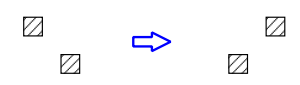

Fig. 4. Switching components and switching operation: type 2 (left) and type 1 (right)

We shall denote by $G_{J}$ the switching graph for the solution set with constraint $J$, and simply call it the $J$-constraint switching graph. If distinction is preferable, we shall call the switching graph $G$ of all the solutions without constraint the full switching graph and further add the projection data like $G[f]$ or $G_{J}[f]$. It is obvious from the definition that for any $J$ (not necessarily unique) $G_{J}$ becomes a full subgraph of $G$. Although $G$ is known to be connected by Ryser's theorem, it is not obvious if $G_{J}$ is connected, too. We shall first establish this.

Theorem 2. Let $J$ be a prohibited region which is a unique figure. Then the $J$ constraint switching graph $G_{J}$ is a connected full subgraph of the full switching graph $G$. 
Proof. The prohibited region $J$ is constructed step by step, by adding a cell each time from left and from bottom, so that

$$
\emptyset=J_{0} \subset J_{1} \subset J_{2} \subset \cdots \subset J_{m}=J,
$$

where each $J_{k}$ consists of $k$ cells in the renormalized form, that is, consists of subrectangles with the bottom edge on the $x$-axis and with decreasing heights. The corresponding constraint switching graphs $G_{J_{k}}$ constitute a decreasing sequence of full subgraphs

$$
G=G_{J_{0}} \supset G_{J_{1}} \supset G_{J_{2}} \supset \cdots \supset G_{J_{m}}=G_{J} .
$$

Assume that $G_{J}$ is not connected. Since $G$ is connected, there exists the minimal $k$ such that $G_{j}, j=0,1, \ldots, k$ is connected but $G_{k+1}$ is not. Note that $J_{k+1}=$ $J_{k} \cup\{P\}$, for some cell $P$. Let $A, B$ be two vertices of $G_{J_{k+1}}$ which are connected by a path $\gamma$ in $G_{J_{k}}$, but never in $G_{J_{k+1}}$. We can assume that the other vertices of $\gamma$ are not in $G_{J_{k+1}}$, and moreover, $\gamma$ is the shortest among such paths. These vertices are obtained from $A$ by several switching operations employing the cells of $A$ and using the place or cell at $P$. Since the region to the left and below $P$ are totally contained in $J_{k}$, the cells of $A$ participating in the switching operations together with $P$ must lie to the right and above $P$. Thus in observing what happens along the path $\gamma$ we can restrict our consideration inside this rectangle. (There may be cells below $P$ and to the right of $J_{k}$, or above $J_{k}$ and to the left of $P$. But these do not mutually switch. Hence the argument below is not essentially affected by these cells.) This means that we can only consider the case where $J$ consists of a single cell to the leftmost and the lowest place, which we shall denote by $J$ by abbreviation of notation, just like the initial step of an induction argument.

Note also that since $J$ is in the prohibited region, the figure $A$ does not have a cell at $J$. By the same reason, the figures corresponding to the vertices of $\gamma$ other than the endpoints should all have a cell at $J$. In fact, if there exists a vertex $C$ in the midst, which does not contain $J$ in its cell, then it is a due vertex of $G_{J_{k+1}}$, hence either of the subpaths $\overline{A C}$ or $\overline{C B}$ would be a path shorter than $\gamma$ and connecting two vertices in $G_{J_{k}}$ which are disjoint in $G_{J_{k+1}}$. This violates the choice of $\gamma$. Just by the same reason, vertices not adjacent to the endpoints of $\gamma$ do not contain any cell which constitute a switching component with $J$.

Thus we assume hereafter that $J$ is the left-lower corner cell of $I$ and show a contradiction, assuming that $\gamma$ is a minimal path connecting two vertices $A, Z$ of $G_{1}$ in $G$. The first edge of $\gamma$ corresponds to the switching of $P, Q \in A$ bringing $P$ to the hole $J$, and $Q$ to some vacant place $Q^{\prime}$ in $I \backslash J$, thus producing a new figure $B$, the second vertex of $\gamma$. First note that

(0) $Z$ can never be the next vertex of $B$.

In fact, if so, $J$ must switch with another cell $R$, as in Figure 5 , But if position $U$ is vacant in $A$, then we can execute this modification without using the position $J$, namely as a path in $G_{1}$, by the series of switchings $Q-R, P-U$. On the other 
hand, if $U$ is occupied, then the same modification is also realized as a path in $G_{1}$ by the switchings $P-U, Q-R$. Thus the vertex $C$ next to $B$ should be in $G \backslash G_{1}$. The passage from $B$ to $C$, or more generally, any inner edge of $\gamma$,

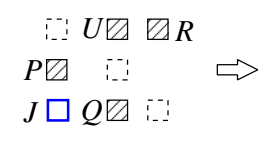

A

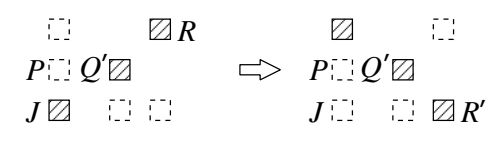

$B$

Fig. 5. Case where the length of $\gamma$ is 2

never corresponds to the switching of cells independent of those touched before, because otherwise, that switching could be preprocessed before the first edge, thus shortening $\gamma$. Hence the second edge $B$ to $C$ corresponds to either of

(i) the switching of $Q^{\prime}$ with another cell $R \in I \backslash J$ (see Figure 6 upper),

(ii) the switching of a new pair $R, S$ in $I \backslash J$ executed using the vacant place after $P$ or $Q$ moved.

Since the essence of the problem does not change by the reflection with respect to the diagonal passing through $J$, we can assume without loss of generality that it is the place of $Q$ which is used in (ii), as in Figure 6 lower.

\begin{tabular}{|c|c|c|c|}
\hline$V \boxminus \quad: \quad \square R$ & $\square R$ & & $Q^{\prime \prime} \square$ \\
\hline$P \square \quad \begin{array}{lll}1 & \Rightarrow\end{array}$ & $P^{\prime} Q^{\prime} \square$ & $\Rightarrow$ & $P^{\prime} \quad: \square R^{\prime}$ \\
\hline$J \square Q \bigotimes \bigotimes U$ & $J$ 团 & & $J \boxminus$ \\
\hline$A$ & $B$ & & $C$ \\
\hline$R \boxminus$ & $R \otimes$ & & $\llbracket S^{\prime}$ \\
\hline$P \square \quad \square \quad \Rightarrow$ & $P^{\prime-1} Q^{\prime}$ & $\Rightarrow$ & $P_{-1}^{\prime} Q^{\prime} \square$ \\
\hline$J \square Q \bigotimes \square S$ & $J \square \square S$ & & $J \bigotimes R^{\prime} \otimes$ \\
\hline$A$ & $B$ & & $C$ \\
\hline
\end{tabular}

Fig. 6. Path $A-B-C$; upper: case (i), lower: case (ii) (only concerned cells are shown)

Let us consider case (i). Note that $R$ can never be in the same column as $P$. In fact, if so, we may directly switch $R$ with $Q$, obtaining the same figure $C$, thus shortening $\gamma$. Hence the above Figure 6 represents the general situation. Next note that the places denoted $U, V$ in $A$ are both occupied by the cells of $A$. In fact, assume e.g. that $U$ is vacant. Then, we can preprocess the switching of $Q$ and $R$, to $Q^{\prime \prime}$ and $U$. Then the above portion of $\gamma$ is shortened to one edge corresponding to the switching of $P$ and $U$. The same is true of $V$. Now we have to consider the next edge. Assume first that it ends at $Z$. Then a new cell $S$ switches with $J$. In this last figure, however, we can see the switchable 


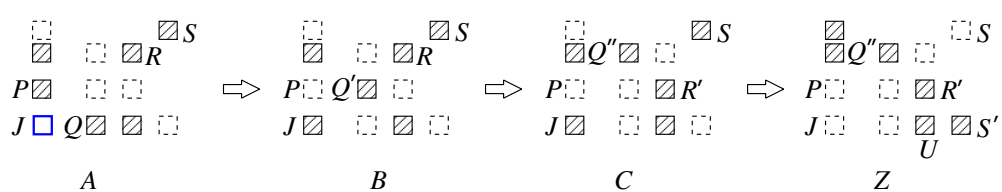

Fig. 7. Case (i) where $\gamma=$ path $A-B-C-Z$

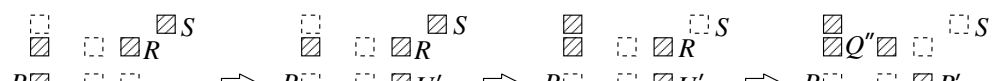

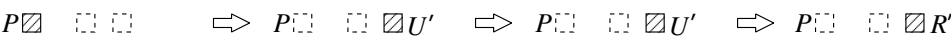

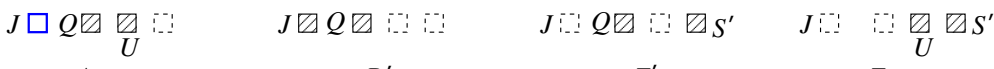

$$
\begin{aligned}
& \text { A } \\
& B^{\prime}
\end{aligned}
$$

Fig. 8. Shortened path $A-B^{\prime}-Z^{\prime}-Z$

pair $Q^{\prime \prime}-U$. After switching this, we obtain another figure $Z^{\prime}$ contained in $G_{1}$, which can be connected with $A$ by a shorter path as in Figure 8 . This contradicts the minimality of $\gamma$. There is a case where $S$ is in the same row as $P$, but the conclusion is the same.

Thus the above path continues to one more inner vertex $A-B-C-D$ with $D \in G \backslash G_{1}$. We can show in this way that we can never reach the end vertex $Z$. See [8] for the detailed proof. The case (ii) can be discussed similarly.

Remark 2. When the prohibited region $J$ is not unique, the connectivity of $G_{J}$ is not necessarily assured. Figure 9 presents such an example. There $G_{J}$ consists of the two shadowed vertices to the right at the top and the bottom of $G$.
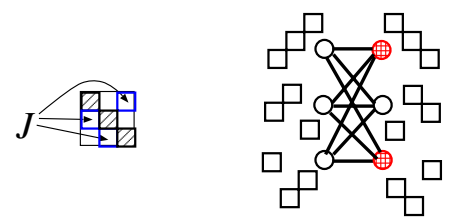

Fig. 9. Example of disconnected constraint switching graph

\section{Experiments}

We apply our construction to discretized slant ellipse. Figure 10 is the original figure. Figure 10b is the Ryser-Kaori reconstruction without constraint, and Figure 10, the type 2 to 1 modification. The value indicated in each figure denotes that of the standard weight function introduced in [6], which increases by the type modification:

$$
w(F):=\sum_{C_{i j} \subset F} i j, \quad \text { where } C_{i j}=[i, i+1) \times[j, j+1) .
$$


If we apply the combination of randomized regression and type 2 to 1 modification, we soon fall in a strong local maximum as in Figure 10d hard to get rid of. On the other hand, if we apply our algorithm with prohibited region $J$ as in Figure 10, we obtain Figure 10, The type 2 to 1 modification with $J$-constraint gives Figure 10f. This time, a number of regression and type 2 to 1 modification with $J$-constraint easily regains the original figure as shown in 10 s.

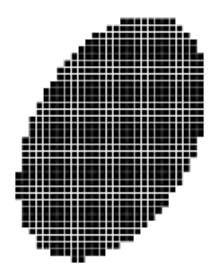

a. 174074

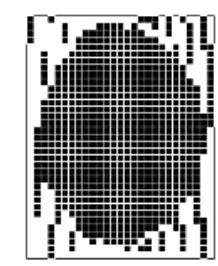

b. 160008

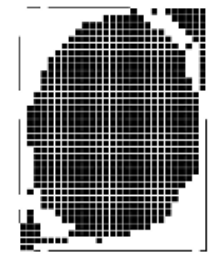

c. 168356

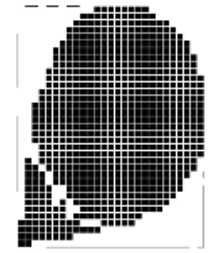

d. 168735

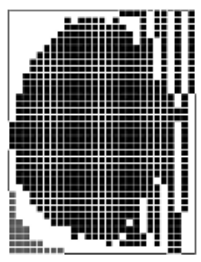

e. 159944

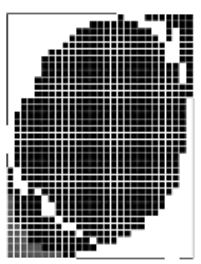

f. 170293

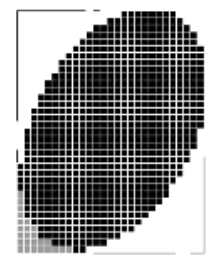

g. 174074

Fig. 10. Reconstruction of slant ellipse

\section{Condition for Prohibited Region}

In the above experiment, we have set a prohibited region from the known original figure. It is, however, desirable that we can set such a region only on the knowledge of the projection data. We therefore examine here a necessary and sufficient condition for $J$ which allows at least one reconstruction for the given projection data. In this section we treat general subsets as prohibited region and do not necessarily require their uniqueness.

Lemma 2. Let $J$ be unique, and let $f=\left\{f_{y}(x), f_{x}(y)\right\}, f^{\prime}=\left\{f_{y}^{\prime}(x), f_{x}^{\prime}(y)\right\}$ be two pairs of J-consistent projection data. Assume that

$$
\forall x \quad f_{y}(x) \leq f_{y}^{\prime}(x), \quad \forall y \quad f_{x}(y) \leq f_{x}^{\prime}(y), \quad\left\|f_{y}^{\prime}-f_{y}\right\|_{L^{1}}=1, \quad\left\|f_{x}^{\prime}-f_{x}\right\|_{L^{1}}=1,
$$

that is, they are different only by one cell. Then there exist reconstruction $F$ from $f$ and $F^{\prime}$ from $f^{\prime}$, each with $J$-constraint, such that the Hamming distance of $F, F^{\prime}$ is equal to 1 , that is, differing only by one cell.

Proof. We shall assume that the columns and rows are so arranged that $J$ has the renormalized form. We proceed by induction on the size $n$ of $f$. For $n=0$, the assertion is trivial. Let it be true for any $J$ and for any $J$-consistent projection 
data, where $f$ has up to $n-1$ cells, and consider the case of $n$ cells. We apply the modified Ryser-Kaori algorithm for reconstruction with $J$ constraint. Let us take the tallest column from $f_{y}(x)$ in the range of $J_{1}$. By permutation of these columns, we can assume without loss of generality that this column is leftmost and that it is the tallest of $f_{y}^{\prime}(x)$ among $J_{1}$, too. In fact, if this is not the case, it means that the cell was added to another column of $f_{y}(x)$ of the same height in the range of $J_{1}$. Then we could take this latter column from the beginning. Now process the lowest cell of this column by the modified Ryser-Kaori algorithm and stop. This should produce the same cell $P$ outside $J$ to the reconstruction figure. By the permutation of rows above $J_{1}$, we can assume without loss of generality that $P$ is just the next cell above the leftmost column of $J_{1}$, hence $J \cup\{P\}$ is still a unique figure. Since this algorithm can be continued to finally produce a legitimate solution with $J$ constraint for each projection data, the remaining projection data should be $J \cup\{P\}$-consistent, and have $n-1$, resp. $n$ cells. Thus by the induction hypothesis, these should have at least one reconstruction $F_{0}$, $F_{0}^{\prime}$ with $J \cup\{P\}$-constraint of Hamming distance $=1$. Then $F=F_{0} \cup\{P\}$, $F^{\prime}=F_{0}^{\prime} \cup\{P\}$ will be a desired pair of reconstructions with $J$-constraint.

Lemma 3. Let $f=\left\{f_{y}(x), f_{x}(y)\right\}$ be a consistent pair of projection data. Then for a cell $P$, the projection data augmented by $P, f+\operatorname{proj}(P)$, is consistent if and only if there exists a reconstruction $F$ for $f$ for which the place $P$ is vacant.

Proof. The sufficiency is obvious: if $f$ admits a reconstruction $F$ for which the place $P$ is vacant, then, the augmented projection data will be those for a valid figure $F \cup\{P\}$. Let us prove the necessity. Assume that the augmented projection data is consistent. Then there are reconstructions for the augmented data. If there is one $F^{\prime}$ among them for which the place $P$ is filled, then, $F=F^{\prime} \backslash\{P\}$ will be a reconstruction for the original data with the place $P$ vacant. Thus assume that the place $P$ is always vacant in any reconstruction $F^{\prime}$ for the augmented projection data and nevertheless that $P$ is always present in any reconstruction $F$ for the original data. Then the Hamming distance of $F$ and $F^{\prime}$ gains one at $P$, and at least 2 outside $P$, because $|F \backslash\{P\}|=n-1$ and $\left|F^{\prime} \backslash\{P\}\right|=\left|F^{\prime}\right|=n+1$. Thus it is $\geq 3$, contradicting Lemma 2 (applied with $J=\emptyset)$.

Employing Lemma 3 repeatedly, we can add a cell at any place as well as the corresponding augmentation of the projection data keeps the consistency of LorentzRyser. Thus we can finally reach a unique figure. But this does not imply that we can adopt the place of thus added cells as the constraint set. We have, however, the following criterion for prohibited region which is verifiable only from the projection data:

Theorem 3. Let $f=\left\{f_{y}(x), f_{x}(y)\right\}$ be a consistent projection data. A region $J$ (not necessarily unique) can be set as a prohibited region to the data $f$ if the augmented projection data $f+\operatorname{proj}(J)$ satisfies the consistency condition of Lorentz-Ryser, and if $J$ is incrementally constructed cell by cell in such a way that each column is filled until it is maximal satisfying the consistency. Any (not necessarily unique) subset of a set constructed in this way is again an admissible 
prohibited region. Especially, we can construct a subregion $J$ such that $I \backslash J$ is a reconstruction from $f$.

Thus a subregion of such $J$ consisting of rectangles in the renormalized form can serve for our discussion hitherto.

Proof. Since the order of adding cells is inessential, we can assume without loss of generality, that we add cells starting from the leftmost column and proceed upward, verifying the consistency cell by cell. In view of Lemma 3 the first cell can be added if and only if $f$ admits a reconstruction with this cell vacant. Hence this cell certainly constitute a part of $J$. Assume that we proceed in the first column and successfully added $J_{0 k}=\bigcup_{j=0}^{k} C_{0 j}$, where $C_{0 j}=[0,1) \times[j, j+1)$, having a reconstruction from $f+\operatorname{proj}\left(J_{0 k}\right)$ with these cells filled. The next cell $C_{0, k+1}$ can be added, again in view of Lemma 3 if there exists a reconstruction from $f+\operatorname{proj}\left(J_{0 k}\right)$ with this cell vacant. But this may have also vacant place among $J_{0 k}$. We claim that nevertheless there is a reconstruction with $J_{0 k}$ filled and with $C_{0, k+1}$ vacant. This is not obvious, but can be proved by an elementary argument similar to that of Theorem 2. We omit the details. If on the other hand, $C_{0, k+1}$ cannot be added, it is filled for all reconstructions from $f+\operatorname{proj}\left(J_{0 k}\right)$, hence especially for those with $J_{0 k}$ filled. Thus we proceed to the next upper cell. Continuing this, we finally fill whole the first column with original and added cells. Therefore this column does not concern the problem hereafter, and the argument goes just in the same way with the next column.

Though the cell-handling order can be arbitrary, a well arranged one is preferable to obtain a better figure. In Figure 11 we show two examples against the projection data of Figure 10 a. In each figure, the black region shows $J$ and the complement presents a reconstruction canonical in some sense.

Remark 3. We cannot omit the assumption of maximality of $J$ in the above theorem. Actually we have a counter-example as in Figure 12. This shows in the
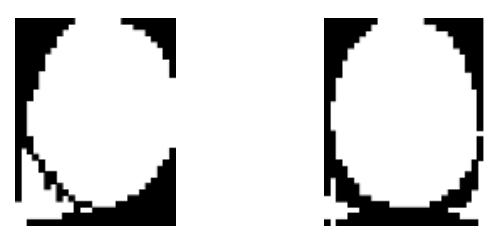

Fig. 11. Reconstructions of slant ellipse with maximal prohibited region; left: from leftmost column; right: from the highest column

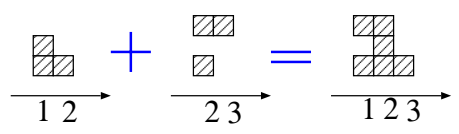

Fig. 12. Counter example without the assumption of maximality 
same time that the assertion "if $f, f^{\prime}$ are two pairs of consistent projection data and $f \cup f^{\prime}$ is also consistent, then there exist reconstructions $F, F^{\prime}$ of $f, f^{\prime}$ such that $F \cap F^{\prime}=\emptyset "$ is false even if all projections are unique.

In general, it is difficult to find the relation between switching graphs $G[f]$ and $G[f \cup \operatorname{proj}(\mathrm{J})]$. We now see, however, that they are connected through the common connected full subgraph $G_{J}[f]$.

\section{References}

1. Anstee, R. P.: Properties of a class of $(0,1)$-matrices covering a given matrix. Canadian J. Math. 34 (1982) $438-453$

2. Brualdi, R. A. : Matrices of zeros and ones with fixed row and column sum vectors. Linear Alg. and Appl. 33 (1980) 159 - 231

3. Brualdi, R. A., Dahl, G.:Matrices of zeros and ones with given line sums and a zero block. Linear Alg. and Appl. 371 (2003) 191 - 207

4. Dahl, G., Brualdi, R.A.: Matrices of zeros and ones with given line sums. Electronic Notes in Discrete Mathematics 20 (2005) 83-97

5. Fulkerson, D. R.: Zero-one matrices with zero trace. Pacific J. Math. 10 (1960) 831 $-836$

6. Kaneko, A., Huang, L.: Reconstruction of plane figures from two projections. In: Herman, T. G., Kuba, A. (eds.): Discrete Tomography. Birkhauser (1999) 115-135

7. Kaneko A., Nagahama, R.: Switching graphs and digraphs associated with total reconstructed sets from two projection data. Nat. Sci. Report Ochanomizu Univ. 56-2 (2005) 33-45

8. Kaneko A., Nagahama, R.: Reconstruction from Two Projections with Prohibited Subregion - Algorithm, Switching Graph and Consistency. Tech. Rep. Dep. Info. Sci. 06-2 May (2006)

9. Kong, T. Y., Herman, T. G.: Tomographic equivalence and switching operations. In: Herman, T. G., Kuba, A. (eds.): Discrete Tomography. Birkhauser (1999) 59 84

10. Kuba, A.: Reconstruction of unique binary matrices with prescribed elements. Acta Cybernetica 12 (1995) 57-70

11. Kuba, A., Herman, T. G.: Discrete tomography: a historical overview. In: Herman, T. G., Kuba, A. (eds.): Discrete Tomography. Birkhauser (1999) $3-34$ 